\section{Appréhender le changement climatique dans une interface Homme-forêt}

La sauvegarde des forêts tropicales est une problématique déjà relativement ancienne qui est en train d'être supplantée par la question du changement climatique et ses impacts forestiers. Plus précisément, à l'heure actuelle, presque toutes les actions de grande portée en faveur des forêts tropicales sont présentées dans un contexte de changement climatique. Effet de mode, moyen de réorienter les flux financiers ou opportunité de relancer la question des forêts ? On préférera y voir une opportunité bienvenue de donner, grâce à la foresterie du carbone, un nouveau souffle à la réflexion sur l'aménagement et la gestion des forêts tropicales.

Peut-être est-il utile d'expliquer ce que I'on peut qualifier de nouveau souffle. Durant quelques dizaines d'années, à savoir la période 1950-1980, les relations Nord-Sud en matière de coopération au développement et de recherche scientifique dans le domaine forestier ont été marquées essentiellement par les apports techniques, le reboisement sous toutes ses formes servant de guide. La période qui a succédé est caractérisée par l'émergence de mots-clés comme la participation, la biodiversité, le développement équilibré homme-femme, I'autonomisation (empowerment), la gouvernance, et maintenant - nous en sommes les contemporains - le changement climatique. Ces mots-clés illustrent des infléchissements, voire des changements au niveau des politiques de développement. Les résultats obtenus sont-ils à la hauteur ? Le doute est permis ... Quelques exemples : (i) après 50 à 60 ans de relations forestières Nord-Sud postcoloniales, il est difficile d'évoquer des progrès significatifs en matière de gestion durable des forêts tropicales ; la part « illégale » de l'exploitation des bois tropicaux ne diminue pas; (ii) des dizaines d'années d'efforts en faveur de la participation et souvent contre l'autorité de l'Etat (les deux approches étant souvent non seulement concomitantes, mais liées) n'ont pas mené à des améliorations concrètes et à grande échelle en ce qui concerne la dévolution de droits d'usage aux populations locales ; (iii) la destruction et la dégradation des forêts tropicales ralentissent à peine ; il suffit pour s'en convaincre d'étudier les publications quinquennales de la FAO sur I'état des forêts dans le monde ; (iv) dans le même registre, il serait intéressant d'examiner si la situation de pauvreté des populations péri-forestières s'est améliorée, depuis toutes ces d'années !

Pourtant, nombre d'exemples concrets et, surtout, les résultats de la recherche scientifique montrent régulièrement et de manière probante, que la réflexion sur l'avenir des forêts ne peut être dissociée de l'intérêt des populations locales. À I'heure de I'omniprésence du changement climatique dans la discussion, menée de manière de plus en plus critique (Caramel 2013), le moment est venu de relancer, de revaloriser et, sans doute, de réimaginer la participation des populations à l'aménagement et à la gestion des forêts tropicales.

Le propos de cet éditorial tire largement parti de plusieurs recherches entreprises ces dernières années à Madagascar sur les questions d'aménagement et de gestion des forêts plaçant, dans toute la mesure du possible, l'Homme au centre de la démarche de recherche afin d'en dégager les éléments constitutifs d'une interface Homme-forêt mieux comprise. Ces programmes ont été réalisés conjointement par le Département Eaux et Forêts de l'École Supérieure des Sciences Agronomiques (ESSA) et l'École Polytechnique Fédérale de Zurich.

Chaque recherche apporte son lot de résultats, d'observations de toute nature et de recommandations. Les résultats de ces travaux sont souvent inédits ; parfois, ils confirment un savoir préexistant. II sera question ici principalement de questions socioéconomiques et socio-politiques.

L'idée d'un programme de recherche sur les fragments forestiers à Madagascar (Urech 2011, Rabenilalana 2011) repose sur le constat qu'une part importante des massifs forestiers ont été détruits ou morcelés, créant des fragments de forêts plus ou moins interconnectés, qui dessinent une mosaïque paysagère. Les fragments de forêts servent (i) de ressource de produits et de services essentiels pour couvrir les besoins des populations locales et (ii) de tremplin pour la préservation de la biodiversité, notamment par une fonction de relais du réseau des massifs encore préservés.

Dans ce contexte, un fragment correspond à toute surface de forêt naturelle d'une superficie inférieure à 500 ha et entourée de terres agricoles ou de jachères, donc non connectée à un massif forestier. Plus encore que les massifs, les fragments ne bénéficient pas d'une protection qui les préserverait d'une disparition complète, sachant que la cause principale de défrichement des forêts réside dans la culture sur brûlis.

Une recherche portant sur le stock de carbone en forêt dense a été menée parallèlement aux travaux précédents, avec des objectifs différents (Rakoto Ratsimba 2011), elle constitue une contribution importante à la définition d'une foresterie du carbone par l'usage de la télédétection en forêt dense humide, dans un contexte de déforestation et de dégradation des ressources forestières. L'auteur relève que dans le cadre de la mise en place d'un système de mesure, rapportage et vérification du carbone (MRV) à l'échelle d'un pays, des études au niveau local demeurent indispensables pour l'observation des niveaux de dégradation. Se focalisant sur la réduction des émissions de carbone, il souligne que la réussite des programmes REDD est étroitement liée avec la question de la propriété du carbone mais également avec l'intégration de la communauté locale dans l'ensemble du processus, notamment en ce qui concerne les droits d'usage ou de propriété.

Dans le Menabe central, de nombreuses recherches ont été menées sur les forêts, les questions sylvicoles notamment, l'exploitation et la transformation du bois ou même l'agroforesterie (Ganzhorn et Sorg 1996), mais peu nombreuses sont celles qui se 
consacrent à des sujets interdisciplinaires à une échelle régionale.

Deux thèses ont été réalisées parallèlement et en étroite concertation. La première par Dirac Ramohavelo (2009) a considéré les ressources du paysage forestier en s'efforçant de dégager les points de vue des habitants des villages dans lesquels les recherches ont été menées ainsi que le rôle des ressources forestières dans les stratégies paysannes. Dans la seconde (Andriambelo 2011), le même paysage a été étudié, mais de manière plus forestière. Le chercheur s'est situé à l'intérieur du massif et a porté son regard vers l'extérieur, partant du constat que dans le but de trouver un équilibre entre la conservation et les pratiques locales, la notion de gestion des ressources forestières a évolué sous l'influence de termes comme participation, décentralisation, droit d'accès et droit de propriété. Sa recherche est basée sur les besoins (domestiques et commerciaux) des paysans par rapport aux ressources ligneuses des forêts qui les entourent ou qu'ils gèrent.

contrairement aux recherches effectuées dans la zone des forêts pluviales, les travaux concernant le Menabe central ne considéraient généralement les forêts secondaires que de manière marginale. D'où un autre travail de recherche (Razafintsalama 2011), basé sur le constat que si les forêts secondaires du Menabe renferment des biens que la population utilise pour satisfaire ses besoins en produits forestiers, celle-ci accorde plus de valeur d'usage aux forêts secondaires qu'aux forêts intactes. Ainsi, le prélèvement atténue la pression exercée sur les forêts naturelles intactes, concrètement, les stratégies villageoises ont un impact moins important lors de prélèvements des ressources forestières (culture sur brûlis, pâturage, collecte de produits).

Cette analyse des recommandations formulées et des observations reproduites dans plusieurs thèses de doctorat réalisées ces dernières années à Madagascar permet de tirer les conclusions suivantes : (i) L'interface Homme-forêt s'inscrit dans une relation souvent complexe, beaucoup plus complexe qu'il n'apparaît au premier abord, entre les communautés humaines et les ressources naturelles renouvelables. (ii) L'action de I'Homme est souvent guidée par des impératifs rationnels de gestion, compte tenu des situations écologiques, sociales et économiques données. Les familles paysannes ont une vision intégrée des ressources sur lesquelles elles ont la maîtrise, où une certaine maîtrise. Si les chercheurs mettent régulièrement ce constat en évidence, les responsables des actions de développement et les administrations en tiennent assez peu compte. (iii) En extension du point cidessus, il est possible de suggérer, globalement, que les populations péri-forestières agissent de manière souvent plus durable que les autorités gouvernementales, les services administratifs et même les organisations de protection de la nature. Ces populations agissent dans les massifs, dans les fragments ainsi que dans les forêts secondaires. (iv) L'agriculture est non seulement la principale cause directe de la déforestation, mais elle constitue aussi I'un des moyens, peut-être le seul, pouvant entraîner une amélioration significative des conditions de vie des populations locales. (v) Dans la gestion, les forêts (massifs, fragments et forêts secondaires) ne doivent pas être prises en compte séparément, mais considérées comme un élément constitutif du paysage et des moyens d'existence de la population. (vi) À ce jour, les modèles de participation à l'aménagement et à la gestion des ressources forestières n'ont pas amélioré de façon significative l'état des forêts et les conditions de vie de la population. Il est même possible de postuler, comme l'ont suggéré Urech et ses collabora- teurs (2015) en appliquant à Madagascar les critères de réussite de la gestion forestière par les communautés locales proposés par Ostrom (1999), que les conditions d’une démarche participative ne sont pas données à I'heure actuelle. (vii) La problématique du changement climatique se superpose depuis plusieurs années à la question de l'aménagement durable des forêts. Des potentialités nouvelles pourraient s'en dégager, mais présupposent que la distribution des revenus ait lieu de manière équitable entre les différents groupes d'acteurs et que notamment les plus faibles (en général les familles paysannes des confins de la forêt) ne soient en rien lésés

Ces conclusions mènent logiquement à proposer une redéfinition des modalités d'aménagement et de gestion des ressources forestières, impliquant l'État, les administrations ainsi que les appuis extérieurs. Les pistes ci-dessous sont suggérées : (i) La participation des populations locales à l'aménagement et à la gestion des ressources naturelles, des paysages, des terroirs constitue un objectif central de la gestion du patrimoine, au sens large de ce terme. Les difficultés que les populations rurales rencontrent en ce qui concerne la productivité agricole, l'accès au marché, l'accès aux connaissances, les droits d'usage et de propriété, la reconnaissance politique, leur système de valeurs doivent être pris en compte dans le cadre de processus participatifs renouvelés, transparents et équitables. (ii) II convient d'opérer une distinction claire entre aménagement et gestion. L'aménagement est la planification de la mise en valeur de ressources pour répondre à des besoins actuels tout en préservant la capacité de production future. La gestion est la mise en œuvre de l'aménagement par des mesures d'entretien, de récolte, de reconstitution et d'amélioration du potentiel de production. La participation débute avec l'aménagement et ne peut pas se limiter aux modalités de gestion. Qui peut se targuer de n'être jamais intervenu auprès d'une population donnée avec des idées préconçues quant à la manière d'aménager une ressource ? C'est une chose que de disposer de compétences et de moyens liés à ces compétences c'en est une autre que d'imposer des points de vue. (iii) Un séminaire organisé à I'ESSA en 2008 a mis en évidence qu'en ce qui concerne la gestion contractualisée des forêts, I'une des causes expliquant l'hétérogénéité des approches et les difficultés rencontrées par et avec les populations locales réside dans la diversité des conceptions ou des philosophies véhiculées par les intervenants-facilitateurs et dans leur fréquente incapacité à en faire abstraction. II en découle une grande difficulté pour les services administratifs à assurer un minimum de coordination, en d'autres termes à garantir la haute surveillance qui leur incombe. Les intervenants-facilitateurs doivent être mieux encadrés par les services administratifs et agir selon des normes d'intervention uniformisées. (iv) La participation "de tous les acteurs », comme il en est de plus en plus souvent fait état, est un leurre. Un statut prioritaire doit être reconnu et appliqué dans les faits aux populations locales, celles qui sont directement concernées par l'aménagement et la gestion de ressources. (v) II n'est plus possible, aujourd'hui, de faire l'impasse sur l'approche paysage ou écosystémique en intégrant climat, géologie et sols, relief, habitants et formes d'habitat, ressources et modes de mise en valeur dans les questions d'aménagement. La notion de paysage, dans le sens qui lui est donné ici, a beaucoup de similitude avec celle de terroir. RappeIons que le terroir est le lieu de vie et d'activité d'une communauté donnée, en règle générale rurale. Un terroir présente une géométrie variable et des intensités de gestion différentes selon 
les ressources considérées. (vi) Un grand ministère regroupant I'agriculture, l'élevage, l'agroforesterie, la foresterie, les ressources de l'eau, la protection de la nature, disposerait de l'autorité nécessaire pour coordonner l'aménagement des ressources naturelles et exercer la haute surveillance. Cette idée, relativement ancienne, est peu appréciée des forestiers, qui craignent d'être phagocytés par l'agriculture. (vii) Exercer une haute surveillance signifie disposer d'un cadre légal et réglementaire et d'en assurer la mise en œuvre. II faut du personnel formé, des moyens et un appui politique. Les universités peuvent constituer la cheville ouvrière de la démarche. (viii) La foresterie du carbone se superpose à la problématique de la participation au sens large de ce terme et en occulte souvent les difficultés. Le changement climatique et la foresterie du carbone peuvent ouvrir la voie à des approches plus sociales, plus équitables, en un mot plus prometteuses de l'aménagement et de la gestion des ressources naturelles. Dans ce but, l'intérêt des populations locales doit figurer en tête des préoccupations.

\section{Jean-Pierre Sorg}

Ex-Groupe de foresterie pour le développement

Swiss Federal Institute of Technology, Zurich

Switzerland
Urech, Z. L., Zaehringer, J. G., Rickenbach, O., Sorg, J.-P. \& Felber, H. R. 2015. Understanding deforestation and forest fragmentation from a livelihood perspective. Madagascar Conservation \& Development 10, 2: 67-76. (doi:10.4314/mcd.v10i2.5)

\section{RÉFÉRENCES}

Andriambelo, L. H. 2011. Critères de Gestion Durable des Ressources Ligneuses du Paysage Forestier du Menabe Central, Madagascar. Thèse de Doctorat en Sciences Agronomiques, Option Eaux et Forêts, Université d'Antananarivo, Antananarivo.166 pp.

Caramel, L. 2013. Forêts tropicales. Les ratés d’un plan de sauvetage. Le Monde 15 novembre 2013: 2-3.

Dirac Ramohavelo, C. 2009. Stratégies Villageoises pour la Gestion des Paysages Forestiers du Menabe Central, Madagascar. Thèse de Doctorat ès Sciences. Ecole Polytechnique Fédérale de Lausanne, Lausanne. 152 pp. Disponible à http://ow.ly/Z4WR2

ESSA (École Supérieure des Sciences Agronomiques). 2008. Les Parties Prenantes de la Gestion Communautaire des Ressources Naturelles: Coopération, Contradictions, Conflits. Actes du Colloque International 1-3 juillet 2008, École Supérieure des Sciences Agronomique, Université d'Antananarivo, Antananarivo. 93 pp. Voir https://essaforets.wordpress.com/akonny-ala-lejournal-forestier-malgache/n31-foret-et-recherche/

Ganzhorn, J. U. \& Sorg, J.-P. (eds.) 1996. Ecology and Economy of a Tropical Dry Forest in Madagascar. Primate Report, special issue 46, Deutsches Primatenzentrum, Göttingen. 382 pp.

Ostrom, E. 1999. Self-governance and forest resources. Occasional Paper 20. ClFOR, Bogor. 15 pp. (doi:10.17528/cifor/000536)

Rabenilalana, F. M. 2011. Fragmentation et dynamique du paysage de la forêt dense humide de basse altitude. Cas de Manompana - Nord-Est de Madagascar. Thèse de doctorat en Sciences Agronomiques, Option Eaux et Forêts, Université d'Antananarivo. Antananarivo. 151 p., annexes

Rakoto Ratsimba, H. 2011. Approche Multi Scalaire de la Déforestation et de la Dégradation : Évaluation du Stock de Carbone dans la Forêt Dense Humide Orientale de Madagascar. Thèse de Doctorat en Sciences Agronomiques, Option Eaux et Forêts, Université d'Antananarivo, Antananarivo. 137 pp., annexes

Razafintsalama, V. 2011. Valeurs, Rôles et Utilisations Villageoises des Forêts Secondaires dans le Paysage Forestier du Menabe Central - Madagascar. Thèse de Doctorat en Sciences Agronomiques, Option Eaux et Forêts, Université d'Antananarivo. Antananarivo, 163 pp., annexes.

Urech, Z. L. 2011. The Role of Forest Fragments in Local Livelihood Systems. The case of the Manompana Corridor, Eastern Madagascar. Dissertation. Eidg. Techn. Hochschule Zurich. Zurich. 143 pp. (doi:10.3929/ethz-a-007109089) 